\title{
Kink Band Formation in High-strength Bulk Metallic Nanolaminates
}

\author{
T. Nizolek ${ }^{1}$, N.A. Mara ${ }^{2}$, R.J. McCabe ${ }^{3}$, J.T. Avallone ${ }^{1}$, I.J. Beyerlein ${ }^{4}$, T.M. Pollock ${ }^{1}$ \\ 1. Materials Department, University of California Santa Barbara, Santa Barbara, CA 93106, USA \\ 2. Institute for Materials Science and Center for Integrated Nanotechnologies, Los Alamos National \\ Laboratory, Los Alamos, NM 87545, USA \\ 3. Material Science and Technology Division, Los Alamos National Laboratory, Los Alamos, NM \\ 87545, USA \\ 4. Theoretical Division, Los Alamos National Laboratory, Los Alamos, NM 87545, USA
}

Advances in severe plastic deformation processing have enabled the production of bulk, high strength $(>1 \mathrm{GPa})$, copper-niobium nanolaminates in volumes suitable for small structural applications. Recent investigations into the deformation behavior of these materials have shown that nanolaminates with sub$100 \mathrm{~nm}$ layer thicknesses deform primarily by kink band formation during layer parallel compression. These kink bands (see Figure 1) consist of regions of material that have been sheared and rotated to form distinct bands of misoriented layers. While a single kink band is shown in Figure 1, these bands can form complex networks of localized deformation (see Figure 2). Despite this pronounced strain localization, no crack formation is detected and further deformation continues at a constant flow stress (as high as $1.2 \mathrm{GPa}$ for $30 \mathrm{~nm}$ material) [1]. In addition to forming during uniaxial compression, kink banding has been observed to occur during a wide variety of mechanical tests and forming operations (such as bending) that involve layer parallel compression.

While kink bands have only recently been observed in metallic nanolaminates, kinking is a well-known phenomenon in plastically anisotropic materials such as fiber composites [2], oriented polymers [3], and even biological materials such as abalone shell and wood [4,5]. The common characteristic of these materials, and a requirement for kink band formation, is mechanical anisotropy. While $\mathrm{Cu}-\mathrm{Nb}$ nanolaminates are composite materials and may be expected to possess anisotropy due to their lamellar microstructure, only nanolaminates with sub-100 nm layers form pronounced kink bands. This observed length scale dependence of kink band formation is unexpected and is not predicted by models developed for kinking in other materials such as fiber composites.

Raising further questions regarding the applicability of existing models for kink band formation is the lack of a pronounced load drop during kinking of metallic nanolaminates [1]. This is in stark contrast to the dramatic loss of load carrying capacity that characterizes kinking in fiber composites. Thus while kinking is appropriately classified as a failure mechanism in fiber composites, kinking in metallic nanolaminates can be considered to be a non-detrimental deformation mechanism.

In order to investigate the origin of the length scale dependence of kinking and to compare kink band formation in metallic nanolaminates to kinking in other systems, in-situ mechanical testing as well as pre-test and post-test microscopy has been conducted. Microstructural analysis of the undeformed material reveals that a pronounced difference in grain morphology exists between the sub-100 nm nanolaminates and the larger layer thickness nanolaminates which do not form kink bands. Compression tests conducted at $45^{\circ}$ to the layered structure confirm that an increase in grain aspect ratio corresponds to a significant increase in plastic anisotropy. For example, while nanolaminates with a layer thickness of $30 \mathrm{~nm}$ and a highly elongated grain morphology exhibit flow stresses in excess of $1200 \mathrm{MPa}$ when 
compressed parallel to the layers, the stress required for layer parallel shear is only $230 \mathrm{MPa}$. From these results it is concluded that the observed length scale dependence of kink band formation is a result of microstructure-driven changes in the level of plastic anisotropy.

The sequence of events leading to kink band formation is investigated using in situ scanning electron microscopy (SEM) compression testing. These tests reveal that kink bands nucleate at stress concentrations and propagate across the specimen, eventually intersecting a free surface. After propagation, continued deformation of the compression specimen is accommodated via broadening of the kink bands. From these tests it is concluded that kinking in metallic nanolaminates occurs in two distinct stages: propagation and broadening. Transmission electron microscopy demonstrates that void formation and layer debonding do not occur during these stages. This indicates that kink band formation in these materials must be treated as a constant volume plastic deformation process, a result which is not consistent with the assumed kinematics in fiber composite model for kinking [2]. Using these insights, we propose modifications to the existing description of kink band formation [6].

\section{References:}

[1] T. Nizolek, et al, Adv. Eng. Mater. (2014) DOI: 10.1002/adem.201400324

[2] B. Budiansky, N.A. Fleck, J.C. Amazigo, J. Mech. Phys. Solids 46 (1998), p. 1637

[3] G.E. Attenburrow, D.C. Bassett, J. Mater. Sci. 14 (1979), p. 2679

[4] R. Menig, et al, Acta Mater. 48 (2000), p. 2383

[5] L. Benabou, Mech. Mater. 42 (2010), p. 335

[6] The authors wish to acknowledge support by the UC Lab Fees Research Program, Award \#238091.

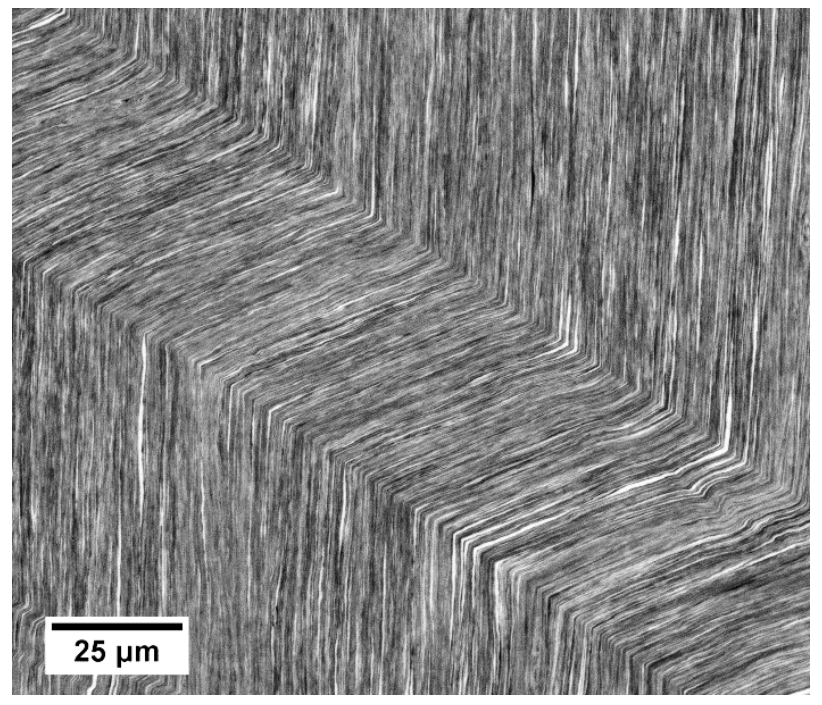

Figure 1. Backscattered SEM image of a kink band in a uniaxial compression specimen of $\mathrm{Cu}-\mathrm{Nb}$ nanolaminate material with $30 \mathrm{~nm}$ nominal layer thickness. The compression direction and the original layer direction are vertical.

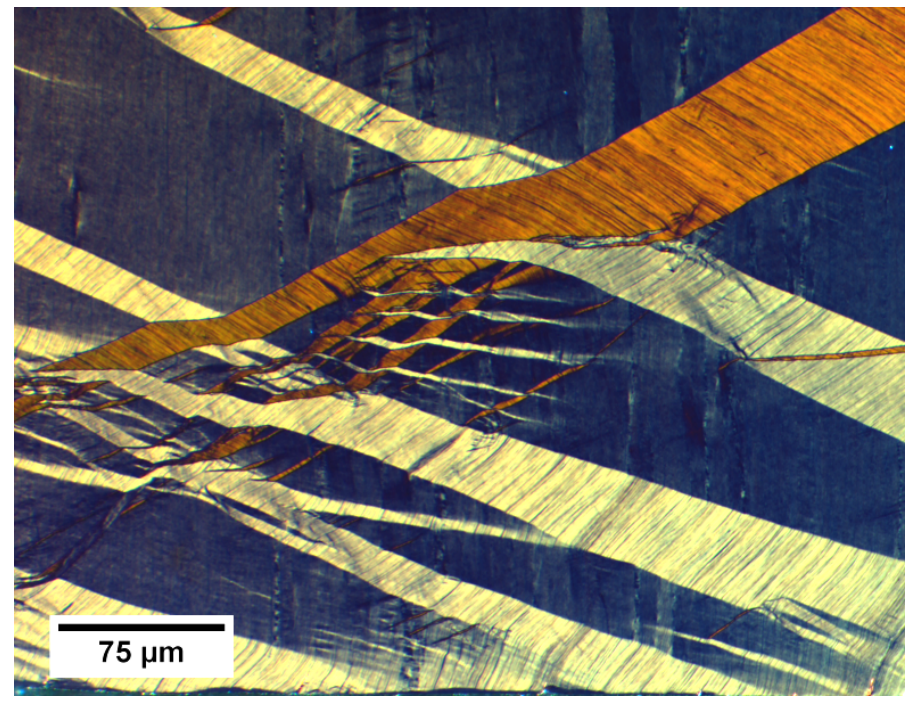

Figure 2. Polarized light micrograph of a complex network of kink bands in a uniaxial compression specimen of $\mathrm{Cu}-\mathrm{Nb}$ nanolaminate material with 15 $\mathrm{nm}$ nominal layer thickness. The compression direction and the original layer direction are vertical. 